\title{
Boundary Interactions for the Semi-Infinite $q$-Boson System and Hyperoctahedral Hall-Littlewood Polynomials ${ }^{\star}$
}

\author{
Jan Felipe VAN DIEJEN and Erdal EMSIZ \\ Facultad de Matemáticas, Pontificia Universidad Católica de Chile, \\ Casilla 306, Correo 22, Santiago, Chile \\ E-mail: diejen@mat.puc.cl, eemsiz@mat.puc.cl
}

Received September 27, 2013, in final form November 26, 2013; Published online December 04, 2013 http://dx.doi.org/10.3842/SIGMA.2013.077

\begin{abstract}
We present a semi-infinite $q$-boson system endowed with a four-parameter boundary interaction. The $n$-particle Hamiltonian is diagonalized by generalized Hall-Littlewood polynomials with hyperoctahedral symmetry that arise as a degeneration of the MacdonaldKoornwinder polynomials and were recently studied in detail by Venkateswaran.
\end{abstract}

Key words: Hall-Littlewood functions; $q$-bosons; boundary fields; hyperoctahedral symmetry

2010 Mathematics Subject Classification: 33D52; 81T25; 81R50; 82B23

\section{Introduction}

The $q$-boson model introduced by Bogoliubov et al. [1] is a quantum many body system on the one-dimensional lattice built of particle creation and annihilation operators representing the $q$ oscillator algebra (cf., e.g., [11, Section 3.1] and [6, Chapter 5] and references therein for further background material concerning the $q$-oscillator algebra and its representations). The model in question can be seen as a limiting case of a more general quantum particle system arising as a $q$ deformation of the totally asymmetric simple exclusion process ( $q$-TASEP) $[2,12,13,14]$. The $n$ particle Bethe ansatz eigenfunctions of the $q$-boson model amount to Hall-Littlewood polynomials, both in the case of a finite periodic lattice (with finite discrete spectrum) $[8,15]$ and in that of an infinite lattice (with bounded absolutely continuous spectrum) [4]. For appropriate boundary fields acting on the particles at the end point of the semi-infinite lattice [5], the Bethe ansatz eigenfunctions result moreover to be given by Macdonald's three-parameter Hall-Littlewood polynomials with hyperoctahedral symmetry associated with the root system $B C_{n}[9, \S 10]$.

Recently it was pointed out that the $B C_{n}$-type Hall-Littlewood polynomials of Macdonald can be viewed as a subfamily of a more general five-parameter family of hyperoctahedral HallLittlewood polynomials that was studied in detail by Venkateswaran [16]; this five-parameter family arises as a $q \rightarrow 0$ degeneration - without parameter confluences - of the MacdonaldKoornwinder multivariate Askey-Wilson polynomials [7, 10]. The purpose of the present note is to show that the five-parameter hyperoctahedral Hall-Littlewood polynomials at issue constitute the eigenfunctions of a semi-infinite $q$-boson model endowed with boundary interactions that involve both the particles at the end point of the lattice and those at its nearest neighboring site. The underlying boundary deformation of the $q$-boson field algebra violates the principle of ultralocality: the particle creation and annihilation operators belonging to the end point and its nearest neighboring site no longer commute, and moreover, the $n$-particle eigenfunctions are only of the usual coordinate Bethe ansatz form away from the end point.

\footnotetext{
${ }^{\star}$ This paper is a contribution to the Special Issue in honor of Anatol Kirillov and Tetsuji Miwa. The full collection is available at http://www.emis.de/journals/SIGMA/InfiniteAnalysis2013.html
} 
Remark 1. To avoid possible confusion, it is important to emphasize that the parameter $q$ of the $q$-boson model does not correspond to the $q$-deformation parameter that enters in Macdonald's theory of orthogonal polynomials associated with root systems [9, 10] but rather to the parameter $t$ used there. A different parameter $t$ is employed below to abbreviate our notation for a frequently appearing product comprised by the four Askey-Wilson-type parameters $t_{1}, \ldots, t_{4}$ of the Macdonald-Koornwinder polynomial (and its $(q \rightarrow 0)$ Hall-Littlewood-type degeneration).

\section{Hyperoctahedral Hall-Littlewood polynomials}

\subsection{Orthogonality}

Let $W$ be the hyperoctahedral group formed by the semi-direct product of the symmetric group $S_{n}$ and the $n$-fold product of the cyclic group $\mathbb{Z}_{2} \cong\{1,-1\}$. An element $w=(\sigma, \epsilon) \in W$ acts naturally on $\xi=\left(\xi_{1}, \ldots, \xi_{n}\right) \in \mathbb{R}^{n}$ via $w \xi:=\left(\epsilon_{1} \xi_{\sigma_{1}}, \ldots, \epsilon_{n} \xi_{\sigma_{n}}\right)$ (with $\sigma \in S_{n}$ and $\epsilon_{j} \in$ $\{1,-1\}$ for $j=1, \ldots, n)$. The algebra $A$ of $W$-invariant polynomials on the torus $\mathbb{T}_{n}:=$ $\mathbb{R}^{n} /\left(2 \pi \mathbb{Z}^{n}\right)$ is spanned by the hyperoctahedral monomial symmetric functions

$$
m_{\lambda}(\xi)=\sum_{\mu \in W \lambda} e^{i\langle\mu, \xi\rangle}, \quad \lambda \in \Lambda_{n}
$$

where $\Lambda_{n}$ stands for the set of partitions $\lambda=\left(\lambda_{1}, \ldots, \lambda_{n}\right) \in \mathbb{Z}^{n}$ with the convention $\lambda_{1} \geq \cdots \geq$ $\lambda_{n} \geq 0$, and the summation is meant over the orbit of $\lambda$ with respect to the action of $W$; the bracket $\langle\cdot, \cdot\rangle$ refers to the standard inner product on $\mathbb{R}^{n}$, i.e. $\langle\mu, \xi\rangle=\mu_{1} \xi_{1}+\cdots+\mu_{n} \xi_{n}$.

The basis of hyperoctahedral Hall-Littlewood polynomials $\mathrm{p}_{\lambda}(\xi), \lambda \in \Lambda_{n}$ studied in [16] arises from the monomial basis via a (partial) Gram-Schmidt-like process as the trigonometric polynomials of the form

$$
\mathrm{p}_{\lambda}(\xi)=m_{\lambda}(\xi)+\sum_{\substack{\mu \in \Lambda_{n} \\ \text { with } \mu<\lambda}} c_{\lambda, \mu} m_{\mu}(\xi), \quad c_{\lambda, \mu} \in \mathbb{C},
$$

such that

$$
\left\langle\mathrm{p}_{\lambda}, m_{\mu}\right\rangle_{\Delta}=0 \quad \text { if } \quad \mu<\lambda
$$

(so $\left\langle\mathrm{p}_{\lambda}, \mathrm{p}_{\mu}\right\rangle_{\Delta}=0$ if $\mu<\lambda$ ). Here we have employed the hyperoctahedral dominance partial ordering of the partitions

$$
\forall \mu, \lambda \in \Lambda_{n}: \quad \mu \leq \lambda \quad \text { iff } \quad \sum_{1 \leq j \leq k} \mu_{j} \leq \sum_{1 \leq j \leq k} \lambda_{j} \quad \text { for } \quad k=1, \ldots, n
$$

(which differs from the usual dominance partial order in that one does not demand the additional degree homogeneity condition $\mu_{1}+\cdots+\mu_{n}=\lambda_{1}+\cdots+\lambda_{n}$ for the partitions to be comparable) together with the following inner product on $A$ :

$$
\langle f, g\rangle_{\Delta}:=\frac{1}{(2 \pi)^{n}|W|} \int_{\mathbb{T}_{n}} f(\xi) \overline{g(\xi)}|\Delta(\xi)|^{2} \mathrm{~d} \xi, \quad f, g \in A,
$$

with $|W|=2^{n} n$ ! denoting the order of the hyperoctahedral group and

$$
\Delta(\xi):=\prod_{1 \leq j<k \leq n} \frac{\left(1-e^{i\left(\xi_{j}-\xi_{k}\right)}\right)\left(1-e^{i\left(\xi_{j}+\xi_{k}\right)}\right)}{\left(1-q e^{i\left(\xi_{j}-\xi_{k}\right)}\right)\left(1-q e^{i\left(\xi_{j}+\xi_{k}\right)}\right)} \prod_{1 \leq j \leq n} \frac{1-e^{2 i \xi_{j}}}{\prod_{r=1}^{4}\left(1-t_{r} e^{i \xi_{j}}\right)} .
$$


Throughout it is assumed that the parameters belong to the domain

$q \in(0,1) \quad$ and $\quad t_{r} \in(-1,1) \backslash\{0\}, \quad r=1, \ldots, 4$.

The hyperoctahedral Hall-Littlewood polynomials satisfy the following orthogonality relations [16]:

$$
\left\langle\mathrm{p}_{\lambda}, \mathrm{p}_{\mu}\right\rangle_{\Delta}= \begin{cases}0 & \text { if } \quad \lambda \neq \mu \\ \mathcal{N}_{\lambda} & \text { if } \quad \lambda=\mu\end{cases}
$$

where

$$
\mathcal{N}_{\lambda}:=\frac{(1-q)^{n}\left(t q^{m_{0}(\lambda)-1}\right)_{m_{0}(\lambda)}}{\left(t q^{2 m_{0}(\lambda)}\right)_{m_{1}(\lambda)} \prod_{1 \leq r<s \leq 4}\left(t_{r} t_{s}\right)_{m_{0}(\lambda)} \prod_{l \geq 0}(q)_{m_{l}(\lambda)}} \quad \text { with } \quad t:=t_{1} t_{2} t_{3} t_{4}
$$

Here the multiplicity $m_{l}(\lambda)$ counts the number of parts $\lambda_{j}, 1 \leq j \leq n$ of size $\lambda_{j}=l$ (so $m_{0}(\lambda)$ is equal to $n$ minus the number of nonzero parts) and we have used $q$-shifted factorials

$$
(x)_{m}:=(1-x)(1-x q) \cdots\left(1-x q^{m-1}\right)
$$

with the convention that $(x)_{0}=1$. Notice that the orthogonality $\left\langle\mathrm{p}_{\lambda}, \mathrm{p}_{\mu}\right\rangle_{\Delta}=0$ for distinct partitions $\lambda$ and $\mu$ is manifest from the defining properties in equations (1) when both weights are comparable in the hyperoctahedral dominance partial ordering (2), whereas for noncomparable partitions the orthogonality is not at all obvious from the above construction.

\subsection{Explicit formula}

The orthogonality relations in equations $(4)$ - which arise as a $(q \rightarrow 0)$ degeneration of wellknown orthogonality relations for the Macdonald-Koornwinder multivariate Askey-Wilson polynomials $[3,7,10]$ - form a two-parameter extension of Macdonald's orthogonality relations for the Hall-Littlewood polynomials associated with the root system $B C_{n}[9, \S 10]$. An explicit formula for the hyperoctahedral Hall-Littlewood polynomials (1) generalizing the corresponding classic formula of Macdonald is given by [16]

$$
\mathrm{p}_{\lambda}(\xi)=\frac{1}{n_{\lambda}} \sum_{w \in W} C_{\lambda}(w \xi) e^{-i\langle\lambda, w \xi\rangle}
$$

with

$$
C_{\lambda}(\xi):=\prod_{1 \leq j<k \leq n} \frac{\left(1-q e^{i\left(\xi_{j}-\xi_{k}\right)}\right)\left(1-q e^{i\left(\xi_{j}+\xi_{k}\right)}\right)}{\left(1-e^{i\left(\xi_{j}-\xi_{k}\right)}\right)\left(1-e^{i\left(\xi_{j}+\xi_{k}\right)}\right)} \prod_{\substack{1 \leq j \leq n \\ \lambda_{j}>0}} \frac{\prod_{r=1}^{4}\left(1-t_{r} e^{i \xi_{j}}\right)}{1-e^{2 i \xi_{j}}}
$$

and

$$
n_{\lambda}:=(1-q)^{-n}(-1)_{m_{0}(\lambda)}\left(t q^{2 m_{0}(\lambda)}\right)_{m_{1}(\lambda)} \prod_{l \geq 0}(q)_{m_{l}(\lambda)}
$$




\subsection{Pieri-type recurrence relation}

The $(q \rightarrow 0)$ degeneration of a Pieri-type recurrence relation for the Macdonald-Koornwinder multivariate Askey-Wilson polynomials [3, Section 6] readily entails a corresponding recurrence relation for the normalized hyperoctahedral Hall-Littlewood polynomials

$$
P_{\lambda}(\xi):=c_{\lambda} \mathrm{p}_{\lambda}(\xi)
$$

where

$$
c_{\lambda}:=\frac{\tau_{1}^{\lambda_{1}} \cdots \tau_{n}^{\lambda_{n}}\left(t q^{2 m_{0}(\lambda)}\right)_{m_{1}(\lambda)} \prod_{l \geq 0}(q)_{m_{l}(\lambda)}}{(q)_{n} \prod_{1<r \leq 4}\left(t_{1} t_{r} q^{m_{0}(\lambda)}\right)_{n-m_{0}(\lambda)}}
$$

with $\tau_{j}:=q^{n-j} t_{1}$ for $j=1, \ldots, n$.

Proposition 1 (Pieri formula). The normalized hyperoctahedral Hall-Littlewood polynomials $P_{\lambda}(\xi), \lambda \in \Lambda_{n}$ satisfy the recurrence relation

$$
\begin{aligned}
P_{\lambda}(\xi) & \sum_{j=1}^{n}\left(2 \cos \left(\xi_{j}\right)-\tau_{j}-\tau_{j}^{-1}\right) \\
\quad= & \sum_{\substack{1 \leq j \leq n \\
\text { s.t. } \lambda+e_{j} \in \Lambda_{n}}} V_{j}^{+}(\lambda)\left(P_{\lambda+e_{j}}(\xi)-P_{\lambda}(\xi)\right)+\sum_{\substack{1 \leq j \leq n \\
\text { s.t. } \lambda-e_{j} \in \Lambda_{n}}} V_{j}^{-}(\lambda)\left(P_{\lambda-e_{j}}(\xi)-P_{\lambda}(\xi)\right),
\end{aligned}
$$

with the vectors $e_{1}, \ldots, e_{n}$ denoting the standard unit basis of $\mathbb{Z}^{n}$ and

$$
\begin{aligned}
V_{j}^{+}(\lambda):= & \tau_{j}^{-1}\left[m_{\lambda_{j}}(\lambda)\right]\left(1-t q^{2 m_{0}(\lambda)+m_{1}(\lambda)-1}\right)^{\delta_{\lambda_{j}-1}+\delta_{\lambda_{j}}} \\
& \times\left(\frac{\prod_{1<r \leq 4}\left(1-t_{1} t_{r} q^{m_{0}(\lambda)-1}\right)}{\left(1-t q^{2 m_{0}(\lambda)-2}\right)\left(1-t q^{2 m_{0}(\lambda)-1}\right)}\right)^{\delta_{\lambda_{j}}}, \\
V_{j}^{-}(\lambda):= & \tau_{j}\left[m_{\lambda_{j}}(\lambda)\right]\left(\frac{\left(1-t q^{m_{0}(\lambda)-1}\right) \prod_{1<r<s \leq 4}\left(1-t_{r} t_{s} q^{m_{0}(\lambda)}\right)}{\left(1-t q^{2 m_{0}(\lambda)-1}\right)\left(1-t q^{2 m_{0}(\lambda)}\right)}\right)^{\delta_{\lambda_{j}-1}} .
\end{aligned}
$$

Here we have employed the $q$-integers $[m]:=\left(1-q^{m}\right) /(1-q)$ for $m=0,1,2,3, \ldots$ as well as the discrete delta function on $\mathbb{Z}: \delta_{l}:=1$ if $l=0$ and $\delta_{l}:=0$ otherwise (and the abbreviation 's.t.' in the conditional sums on the r.h.s. of the recurrence stands for 'such that').

Proof. As a $(q \rightarrow 0)$ degeneration of the principal specialization formula for the MacdonaldKoornwinder polynomials (see, e.g., [3, equations (6.1), (6.18), (6.43a)]) one finds that (assuming momentarily $\left.t_{1}>0\right)$ :

$$
\mathrm{p}_{\lambda}\left(i \log \left(\tau_{1}\right), \ldots, i \log \left(\tau_{n}\right)\right)=\frac{1}{c_{\lambda}}
$$

with $c_{\lambda}$ taken from equation (6b). This implies that the normalization of $P_{\lambda}(\xi)(6)$ is such that the polynomials in question satisfy a $(q \rightarrow 0)$ degeneration of the Pieri-type recurrence formula in equations $(6.4),(6.5),(6.12),(6.13)$ of [3], which - upon performing the limit - produces equation (7). 


\section{Boundary interactions for the semi-infinite $q$-boson system}

\subsection{Deformed $q$-boson field algebra}

Let $\ell^{2}\left(\Lambda_{n}, \mathcal{N}\right)$ be the Hilbert space of functions $f: \Lambda_{n} \rightarrow \mathbb{C}$ determined by the inner product

$$
\langle f, g\rangle_{n}:=\sum_{\lambda \in \Lambda_{n}} f(\lambda) \overline{g(\lambda)} \mathcal{N}_{\lambda}, \quad f, g \in \ell^{2}\left(\Lambda_{n}, \mathcal{N}\right)
$$

with $\mathcal{N}_{\lambda}$ given by equation (4b) and the convention that $\Lambda_{0}:=\{\varnothing\}$ and $\ell^{2}\left(\Lambda_{0}, \mathcal{N}\right):=\mathbb{C}$. We think of $\ell^{2}\left(\Lambda_{n}, \mathcal{N}\right)$ as the Hilbert space for a system of $n$ quantum particles on the nonnegative integer lattice $\mathbb{N}:=\{0,1,2, \ldots\}$ (i.e. the parts $\lambda_{j}, j=1, \ldots, n$ of $\lambda \in \Lambda_{n}$ encode the positions of the particles in question). In the Fock space

$$
\mathcal{H}:=\bigoplus_{n \geq 0} \ell^{2}\left(\Lambda_{n}, \mathcal{N}\right),
$$

consisting of all sequences $\sum_{n \geq 0} f_{n}$ with $f_{n} \in \ell^{2}\left(\Lambda_{n}, \mathcal{N}\right)$ such that $\sum_{n \geq 0}\left\langle f_{n}, f_{n}\right\rangle_{n}<\infty$, we introduce bounded annihilation operators $\beta_{l}, l \in \mathbb{N}$ that are perturbed at the boundary site $\ell=0$ and act on $f \in \ell^{2}\left(\Lambda_{n}, \mathcal{N}\right)$ via

$$
\left(\beta_{l} f\right)(\lambda):=\frac{f\left(\beta_{l}^{*} \lambda\right)}{\left(1-t q^{2 m_{0}(\lambda)+m_{1}(\lambda)}\right)^{\delta_{l}}}, \quad \lambda \in \Lambda_{n-1},
$$

if $n>0$, and $\beta_{l} f:=0$ if $n=0$. Here $\beta_{l}^{*} \lambda \in \Lambda_{n}$ is obtained from $\lambda$ by adding a part of size $l$. The action on $f \in \ell^{2}\left(\Lambda_{n}, \mathcal{N}\right)$ of the adjoint of $\beta_{l}$ in $\mathcal{H}$ produces the creation operator

$$
\begin{aligned}
\left(\beta_{l}^{*} f\right)(\lambda)= & f\left(\beta_{l} \lambda\right)\left[m_{l}(\lambda)\right]\left(1-t q^{2 m_{0}(\lambda)+m_{1}(\lambda)-1}\right)^{\delta_{l}+\delta_{l-1}} \\
& \times\left(\frac{\left(1-t q^{m_{0}(\lambda)-2}\right) \prod_{1 \leq r<s \leq 4}\left(1-t_{r} t_{s} q^{m_{0}(\lambda)-1}\right)}{\left(1-t q^{2 m_{0}(\lambda)-3}\right)\left(1-t q^{2 m_{0}(\lambda)-2}\right)^{2}\left(1-t q^{2 m_{0}(\lambda)-1}\right)}\right)^{\delta_{l}}, \quad \lambda \in \Lambda_{n+1},
\end{aligned}
$$

if $m_{l}(\lambda)>0$, and $\left(\beta_{l}^{*} f\right)(\lambda)=0$ otherwise. Here $\beta_{l} \lambda \in \Lambda_{n}$ is obtained from $\lambda$ with $m_{l}(\lambda)>0$ by discarding a part of size $l$. In the present setting, the role of the number operators is played by the bounded multiplication operators

$$
\left(N_{l} f\right)(\lambda):=q^{m_{l}(\lambda)} f(\lambda), \quad f \in \ell^{2}\left(\Lambda_{n}, \mathcal{N}\right), \quad \lambda \in \Lambda_{n}, \quad l \in \mathbb{N} .
$$

When $t \neq q^{m}$ for $m=1,2,3, \ldots$, the creation and annihilation operators $\beta_{l}^{*}, \beta_{l}$ together with the commuting operators $N_{l},\left(1-t q^{c} N_{0}^{2}\right)^{-1},\left(1-t q^{c} N_{0}^{2} N_{1}\right)^{-1}$ (where $l \in \mathbb{N}$ and $c \in \mathbb{Z}$ ) represent a four-parameter deformation of the $q$-boson field algebra at the boundary sites $l=0$ and $l=1$ :

$$
\begin{aligned}
\beta_{l} N_{k}= & q^{\delta_{l-k}} N_{k} \beta_{l}, \quad \beta_{l}^{*} N_{k}=q^{-\delta_{l-k}} N_{k} \beta_{l}^{*} \\
\beta_{l}^{*} \beta_{l}= & \frac{1-N_{l}}{1-q}\left(1-q^{-1} t N_{0}^{2} N_{1}\right)^{\delta_{l}+\delta_{l-1}} \\
& \times\left(\frac{\left(1-q^{-2} t N_{0}\right) \prod_{1 \leq r<s \leq 4}\left(1-q^{-1} t_{r} t_{s} N_{0}\right)}{\left(1-q^{-3} t N_{0}^{2}\right)\left(1-q^{-2} t N_{0}^{2}\right)^{2}\left(1-q^{-1} t N_{0}^{2}\right)\left(1-q^{-2} t N_{0}^{2} N_{1}\right)}\right) \\
\beta_{l} \beta_{l}^{*}= & \frac{1-q N_{l}}{1-q}\left(1-t N_{0}^{2} N_{1}\right)^{-\delta_{l}+\delta_{l-1}} \times
\end{aligned}
$$




$$
\times\left(\frac{\left(1-q^{-1} t N_{0}\right)\left(1-q t N_{0}^{2} N_{1}\right) \prod_{1 \leq r<s \leq 4}\left(1-t_{r} t_{s} N_{0}\right)}{\left(1-q^{-1} t N_{0}^{2}\right)\left(1-t N_{0}^{2}\right)^{2}\left(1-q t N_{0}^{2}\right)}\right)^{\delta_{l}}
$$

and for $l<k$

$$
\beta_{l} \beta_{k}=\left(\frac{1-q t N_{0}^{2} N_{1}}{1-t N_{0}^{2} N_{1}}\right)^{\delta_{l} \delta_{k-1}} \beta_{k} \beta_{l}, \quad \beta_{l}^{*} \beta_{k}^{*}=\beta_{k}^{*} \beta_{l}^{*}\left(\frac{1-t N_{0}^{2} N_{1}}{1-q t N_{0}^{2} N_{1}}\right)^{\delta_{l} \delta_{k-1}},
$$

and

$$
\beta_{l} \beta_{k}^{*}=\left(\frac{1-q t N_{0}^{2} N_{1}}{1-t N_{0}^{2} N_{1}}\right)^{\delta_{l} \delta_{k-1}} \beta_{k}^{*} \beta_{l}, \quad \beta_{l}^{*} \beta_{k}=\beta_{k} \beta_{l}^{*}\left(\frac{1-t N_{0}^{2} N_{1}}{1-q t N_{0}^{2} N_{1}}\right)^{\delta_{l} \delta_{k-1}} .
$$

Indeed, it is straightforward to verify the commutation relations in equations (11) upon computing the explicit actions of both sides on an arbitrary function $f \in \ell^{2}\left(\Lambda_{n}, \mathcal{N}\right)$ with the aid of the formulas in equations (9) and (10).

\subsection{Hamiltonian}

The Hamiltonian of our semi-infinite $q$-boson system with boundary interaction is of the form

$$
H=V\left(N_{0}, N_{1}\right)+\sum_{l \in \mathbb{N}}\left(\beta_{l}^{*} \beta_{l+1}+\beta_{l+1}^{*} \beta_{l}\right),
$$

where $V\left(N_{0}, N_{1}\right)$ denotes a boundary potential that depends rationally on $N_{0}$ and $N_{1}$. By construction, $H(12)$ preserves the $n$-particle sector $\ell^{2}\left(\Lambda_{n}, \mathcal{N}\right) \subset \mathcal{H}$ and we will denote the restriction of the Hamiltonian to this $n$-particle subspace by $H_{n}$.

Proposition 2 (n-particle Hamiltonian). For any $f \in \ell^{2}\left(\Lambda_{n}, \mathcal{N}\right)$ and $\lambda \in \Lambda_{n}$, one has that

$$
\begin{aligned}
\left(H_{n} f\right)(\lambda)= & V\left(q^{m_{0}(\lambda)}, q^{m_{1}(\lambda)}\right) f(\lambda) \\
& +\sum_{\substack{1 \leq j \leq n \\
\text { s.t. } \lambda+e_{j} \in \Lambda_{n}}} v_{j}^{+}(\lambda) f\left(\lambda+e_{j}\right)+\sum_{\substack{1 \leq j \leq n \\
\text { s.t. } \lambda-e_{j} \in \Lambda_{n}}} v_{j}^{-}(\lambda) f\left(\lambda-e_{j}\right),
\end{aligned}
$$

with

$$
\begin{aligned}
v_{j}^{+}(\lambda):= & {\left[m_{\lambda_{j}}(\lambda)\right]\left(1-t q^{2 m_{0}(\lambda)+m_{1}(\lambda)-1}\right)^{\delta_{\lambda_{j}-1}+\delta_{\lambda_{j}}} } \\
& \times\left(\frac{\left(1-t q^{m_{0}(\lambda)-2}\right) \prod_{1 \leq r<s \leq 4}\left(1-t_{r} t_{s} q^{m_{0}(\lambda)-1}\right)}{\left(1-t q^{2 m_{0}(\lambda)-3}\right)\left(1-t q^{2 m_{0}(\lambda)-2}\right)^{2}\left(1-t q^{\left.2 m_{0}(\lambda)-1\right)}\right.}\right)^{\delta_{\lambda_{j}}}, \\
v_{j}^{-}(\lambda):= & {\left[m_{\lambda_{j}}(\lambda)\right] . }
\end{aligned}
$$

Proof. It is immediate from the explicit actions of $\beta_{l}$ and $\beta_{l}^{*}$ in equations (9) that for any $l \in \mathbb{N}$ : $\left(\beta_{l+1} \beta_{l}^{*} f\right)(\lambda)=0$ if $m_{l}(\lambda)=0$ and

$$
\begin{aligned}
\left(\beta_{l}^{*} \beta_{l+1} f\right)(\lambda)= & {\left[m_{\lambda_{j}}(\lambda)\right]\left(1-t q^{2 m_{0}(\lambda)+m_{1}(\lambda)-1}\right)^{\delta_{l-1}+\delta_{l}} } \\
& \times\left(\frac{\left(1-t q^{m_{0}(\lambda)-2}\right) \prod_{1 \leq r<s \leq 4}\left(1-t_{r} t_{s} q^{m_{0}(\lambda)-1}\right)}{\left(1-t q^{2 m_{0}(\lambda)-3}\right)\left(1-t q^{2 m_{0}(\lambda)-2}\right)^{2}\left(1-t q^{2 m_{0}(\lambda)-1}\right)}\right)^{\delta_{l}} f\left(\beta_{l+1}^{*} \beta_{l} \lambda\right)
\end{aligned}
$$


if $m_{l}(\lambda)>0$, where $\beta_{l+1}^{*} \beta_{l} \lambda=\lambda+e_{j}$ with $j=\min \left\{k \mid \lambda_{k}=l\right\}$ (so $l=\lambda_{j}$ ). Along the same lines it is seen that $\left(\beta_{l+1}^{*} \beta_{l} f\right)(\lambda)=0$ if $m_{l+1}(\lambda)=0$ and

$$
\left(\beta_{l+1}^{*} \beta_{l} f\right)(\lambda)=\left[m_{l+1}(\lambda)\right] f\left(\beta_{l}^{*} \beta_{l+1} \lambda\right)
$$

if $m_{l+1}(\lambda)>0$, where $\beta_{l}^{*} \beta_{l+1} \lambda=\lambda-e_{j}$ with $j=\max \left\{k \mid \lambda_{k}=l+1\right\}$ (so $\left.l=\lambda_{j}-1\right)$. The stated formula thus follows because the boundary potential acts (by definition) via the multiplication $\left(V\left(N_{0}, N_{1}\right) f\right)(\lambda)=V\left(q^{m_{0}(\lambda)}, q^{m_{1}(\lambda)}\right) f(\lambda)$.

\subsection{Diagonalization}

From now on we will pick the boundary potential $V\left(N_{0}, N_{1}\right)$ in $H$ (12) of the form

$$
\begin{aligned}
V\left(N_{0}, N_{1}\right)= & \left(t_{1}^{-1} t N_{0}+t_{1} N_{0}\left(1-\frac{\left(1-q^{-1} t N_{0}\right) \prod_{1<r<s \leq 4}\left(1-t_{r} t_{s} N_{0}\right)}{\left(1-t N_{0}^{2}\right)\left(1-q^{-1} t N_{0}^{2}\right)}\right)\right) \frac{1-N_{1}}{1-q} \\
& +\left(t_{1}+q t_{1}^{-1} N_{0}^{-1}\left(1-\frac{\left(1-q^{-1} t N_{0}^{2} N_{1}\right) \prod_{1<r \leq 4}\left(1-q^{-1} t_{1} t_{r} N_{0}\right)}{\left(1-q^{-2} t N_{0}^{2}\right)\left(1-q^{-1} t N_{0}^{2}\right)}\right)\right) \frac{1-N_{0}}{1-q} .
\end{aligned}
$$

By writing the action of $V\left(N_{0}, N_{1}\right)$ (14) on an arbitrary $f \in \ell^{2}\left(\Lambda_{n}, \mathcal{N}\right)$ as a rational expression in the parameters $t_{r}(r=1, \ldots, 4)$, it is readily seen - upon canceling possible common factors in the numerators and denominators - that $V\left(N_{0}, N_{1}\right)$ constitutes a bounded multiplication operator in $\ell^{2}\left(\Lambda_{n}, \mathcal{N}\right)$. It follows moreover from the Pieri recurrence in Proposition 1 and the explicit formula for $H_{n}$ in Proposition 2 that the Hamiltonian with this boundary potential is diagonalized in the $n$-particle subspace by a hyperoctahedral Hall-Littlewood wave function $\phi_{\xi}: \Lambda_{n} \rightarrow \mathbb{C}$ of the form

$$
\phi_{\xi}(\lambda):=\frac{1}{\mathcal{N}_{\lambda}} \mathrm{p}_{\lambda}(\xi), \quad \lambda \in \Lambda_{n},
$$

where $\xi \in \mathbb{T}_{n}$ plays the role of the spectral parameter.

Proposition 3 (n-particle eigenfunctions). The hyperoctahedral Hall-Littlewood wave function $\phi_{\xi}(15)$ satisfies the eigenvalue equation

$$
H_{n} \phi_{\xi}=E_{n}(\xi) \phi_{\xi} \quad \text { with } \quad E_{n}(\xi):=2 \sum_{1 \leq j \leq n} \cos \left(\xi_{j}\right)
$$

for $H_{n}$ given by equations (13) with $V\left(N_{0}, N_{1}\right)$ taken from equation (14).

Proof. By comparing the normalization of $\phi_{\xi}(\lambda)(15)$ and $P_{\lambda}(\xi)(6)$, one concludes that $\phi_{\xi}(\lambda)=$ $\frac{1}{h_{\lambda}} P_{\lambda}(\xi)$ with

$$
h_{\lambda}=c_{\lambda} \mathcal{N}_{\lambda}=\frac{\tau_{1}^{\lambda_{1}} \cdots \tau_{n}^{\lambda_{n}}\left(t q^{m_{0}(\lambda)-1}\right)_{m_{0}(\lambda)} \prod_{1<r<s \leq 4}\left(t_{r} t_{s} q^{m_{0}(\lambda)}\right)_{n-m_{0}(\lambda)}}{\left(t q^{n-1}\right)_{n}} \mathcal{N}_{0} .
$$

It is thus immediate from equation (7) that

$$
\begin{aligned}
& V\left(q^{m_{0}(\lambda)}, q^{m_{1}(\lambda)}\right) \phi_{\xi}(\lambda)+\sum_{\substack{1 \leq j \leq n \\
\text { s.t. } \lambda+e_{j} \in \Lambda_{n}}} v_{j}^{+}(\lambda) \phi_{\xi}\left(\lambda+e_{j}\right)+\sum_{\substack{1 \leq j \leq n \\
\text { s.t. } \lambda-e_{j} \in \Lambda_{n}}} v_{j}^{-}(\lambda) \phi_{\xi}\left(\lambda-e_{j}\right) \\
& =E_{n}(\xi) \phi_{\xi}(\lambda),
\end{aligned}
$$


with

$$
v_{j}^{+}(\lambda)=V_{j}^{+}(\lambda) \frac{h_{\lambda+e_{j}}}{h_{\lambda}}, \quad v_{j}^{-}(\lambda)=V_{j}^{-}(\lambda) \frac{h_{\lambda-e_{j}}}{h_{\lambda}}
$$

and

$$
V\left(q^{m_{0}(\lambda)}, q^{m_{1}(\lambda)}\right)=\sum_{1 \leq j \leq n}\left(\tau_{j}+\tau_{j}^{-1}\right)-\sum_{\substack{1 \leq j \leq n \\ \text { s.t. } \lambda+e_{j} \in \Lambda_{n}}} V_{j}^{+}(\lambda)-\sum_{\substack{1 \leq j \leq n \\ \text { s.t. } \lambda-e_{j} \in \Lambda_{n}}} V_{j}^{-}(\lambda) .
$$

By plugging in the explicit expressions for $V_{j}^{+}(\lambda), V_{j}^{-}(\lambda)$, and $h_{\lambda}$, and employing the elementary identity

$$
\sum_{1 \leq j \leq n}\left(\tau_{j}+\tau_{j}^{-1}\right)-\sum_{\substack{1 \leq j \leq n \\ \text { s.t. } \lambda+e_{j} \in \Lambda_{n}}} \tau_{j}^{-1}\left[m_{\lambda_{j}}(\lambda)\right]-\sum_{\substack{1 \leq j \leq n \\ \text { s.t. } \lambda-e_{j} \in \Lambda_{n}}} \tau_{j}\left[m_{\lambda_{j}}(\lambda)\right]=t_{1}\left[m_{0}(\lambda)\right]
$$

the coefficients $v_{j}^{+}(\lambda), v_{j}^{-}(\lambda)$ and $V\left(q^{m_{0}(\lambda)}, q^{m_{1}(\lambda)}\right)$ are rewritten in the form given by equations (13b), (13c) and (14).

Remark 2. The diagonalization in Proposition 3 in terms of the hyperoctahedral Hall-Littlewood polynomials implies that our $q$-boson Hamiltonian $H_{n}$ is unitarily equivalent to a multiplication operator governed by the eigenvalue $E_{n}(\xi)(16)$. A complete system of commuting quantum integrals for $H_{n}$ is obtained via this unitary equivalence from the multiplication operators associated with the elements of the algebra $A$ of $W$-invariant trigonometric polynomials on $\mathbb{T}_{n}$. It remains an open problem to present an explicit construction in the spirit of [4] that lifts $H$ (12) with $V\left(N_{0}, N_{1}\right)$ given by equation (14) to an infinite hierarchy of commuting operators in the Fock space $\mathcal{H}(8)$, reproducing the quantum integrals of $H_{n}$ upon restriction to the $n$-particle subspace $\ell^{2}\left(\Lambda_{n}, \mathcal{N}\right)$.

\section{Ultralocality and coordinate Bethe ansatz}

For general parameter values the deformation of the $q$-boson field algebra in Section 3.1 fails to be ultralocal, as the commutativity between the creation and annihilation operators at sites $l=0$ and $l=1$ is broken. The commutativity (and hence ultralocality) is restored when at least one of the four boundary parameters $t_{r}$ tends to zero (so $t \rightarrow 0$ ). It is furthermore clear from the explicit expression in equations (5) for the hyperoctahedral Hall-Littlewood polynomial $\mathrm{p}_{\lambda}(1)$ that the wave function $\phi_{\xi}$ (15) fails to be of the usual coordinate Bethe ansatz form (at the boundary), as the expansion coefficients $C_{\lambda}(w \xi)$ of the plane waves $e^{-i\langle w \xi, \lambda\rangle}$ depend on (the number of nonzero parts of) $\lambda$. By letting at least two of the four boundary parameters $t_{r}$ tend to zero the polynomial $\mathrm{p}_{\lambda}(1)$ reduces to Macdonald's Hall-Littlewood polynomial associated with the root system of type $B C$, which implies that in this limiting case it is possible to rewrite the wave function in the conventional Bethe ansatz form. We end up by detailing our construction for these three- and two-parameter specializations of the boundary interaction.

\subsection{Three-parameter reduction}

When $t_{4} \rightarrow 0$ (so $t \rightarrow 0$ ), the quadratic norm $\mathcal{N}_{\lambda}$ (4b) determining inner product of the Fock space $\mathcal{H}(8)$ simplifies to

$$
\mathcal{N}_{\lambda}=\frac{(1-q)^{n}}{\prod_{1 \leq r<s \leq 3}\left(t_{r} t_{s}\right)_{m_{0}(\lambda)} \prod_{l \geq 0}(q)_{m_{l}(\lambda)}} .
$$


The actions of the annihilation and creation operators $(9)$ on $f \in \ell^{2}\left(\Lambda_{n}, \mathcal{N}\right)$ then reduce to

$$
\left(\beta_{l} f\right)(\lambda)=f\left(\beta_{l}^{*} \lambda\right), \quad \lambda \in \Lambda_{n-1}
$$

with the convention that $\beta_{l} f=0$ if $n=0$, and

$$
\left(\beta_{l}^{*} f\right)(\lambda)=f\left(\beta_{l} \lambda\right)\left[m_{l}(\lambda)\right] \prod_{1 \leq r<s \leq 3}\left(1-t_{r} t_{s} q^{m_{0}(\lambda)-1}\right)^{\delta_{l}}, \quad \lambda \in \Lambda_{n+1}
$$

with the convention that $\left(\beta_{l}^{*} f\right)(\lambda)=0$ if $m_{l}(\lambda)=0$, respectively. Together with the commuting operators $N_{l}(10)$ the creation and annihilation operators in question represent a three-parameter deformation of the $q$-boson field algebra at the boundary site $l=0$ :

$$
\begin{aligned}
& \beta_{l} N_{k}=q^{\delta_{l-k}} N_{k} \beta_{l}, \quad \beta_{l}^{*} N_{k}=q^{-\delta_{l-k}} N_{k} \beta_{l}^{*}, \\
& \beta_{l}^{*} \beta_{l}=\frac{1-N_{l}}{1-q} \prod_{1 \leq r<s \leq 3}\left(1-q^{-1} t_{r} t_{s} N_{0}\right)^{\delta_{l}}, \quad \beta_{l} \beta_{l}^{*}=\frac{1-q N_{l}}{1-q} \prod_{1 \leq r<s \leq 3}\left(1-t_{r} t_{s} N_{0}\right)^{\delta_{l}},
\end{aligned}
$$

preserving the ultralocality:

$$
\beta_{l} \beta_{k}=\beta_{k} \beta_{l}, \quad \beta_{l}^{*} \beta_{k}^{*}=\beta_{k}^{*} \beta_{l}^{*}, \quad \beta_{l} \beta_{k}^{*}=\beta_{k}^{*} \beta_{l}, \quad \beta_{l}^{*} \beta_{k}=\beta_{k} \beta_{l}^{*}
$$

if $l<k$. The corresponding $q$-boson Hamiltonian $H$ (12), with the pertinent reduction of the boundary potential $V\left(N_{0}, N_{1}\right)$ (14) given by

$$
V\left(N_{0}, N_{1}\right)=\left(t_{1}+t_{2}+t_{3}-q^{-1} t_{1} t_{2} t_{3} N_{0}\right)\left(\frac{1-N_{0}}{1-q}\right)+t_{1} t_{2} t_{3} N_{0}^{2}\left(\frac{1-N_{1}}{1-q}\right),
$$

acts on $f$ in the $n$-particle subspace $\ell^{2}\left(\Lambda_{n}, \mathcal{N}\right)$ via

$$
\begin{aligned}
\left(H_{n} f\right)(\lambda)= & \sum_{\substack{1 \leq j \leq n \\
\text { s.t. } \lambda+e_{j} \in \Lambda_{n}}} f\left(\lambda+e_{j}\right)\left[m_{\lambda_{j}}(\lambda)\right] \prod_{1 \leq r<s \leq 3}\left(1-t_{r} t_{s} q^{m_{0}(\lambda)-1}\right)^{\delta_{\lambda_{j}}} \\
& +\sum_{\substack{1 \leq j \leq n \\
\text { s.t. } \lambda-e_{j} \in \Lambda_{n}}} f\left(\lambda-e_{j}\right)\left[m_{\lambda_{j}}(\lambda)\right] \\
& +f(\lambda)\left(\left(t_{1}+t_{2}+t_{3}-q^{-1} t_{1} t_{2} t_{3} q^{m_{0}(\lambda)}\right)\left[m_{0}(\lambda)\right]+t_{1} t_{2} t_{3} q^{2 m_{0}(\lambda)}\left[m_{1}(\lambda)\right]\right) .
\end{aligned}
$$

\subsection{Two-parameter reduction}

From the defining orthogonality and the triangularity properties of the hyperoctahedral HallLittlewood polynomials $\mathrm{p}_{\lambda}, \lambda \in \Lambda_{n}$ detailed in Section 2.1, it is read-off that for $t_{3}, t_{4} \rightarrow 0$ these polynomials reduce to Macdonald's Hall-Littlewood polynomials associated with the $B C$ type root system $[9, \S 10]$. This implies that they can be rewritten in terms of Macdonald's formula:

$$
\mathrm{p}_{\lambda}(\xi)=\mathcal{N}_{\lambda} \sum_{w \in W} C(w \xi) e^{-i\langle\lambda, w \xi\rangle}
$$

with

$$
C(\xi)=\prod_{1 \leq j<k \leq n} \frac{\left(1-q e^{i\left(\xi_{j}-\xi_{k}\right)}\right)\left(1-q e^{i\left(\xi_{j}+\xi_{k}\right)}\right)}{\left(1-e^{i\left(\xi_{j}-\xi_{k}\right)}\right)\left(1-e^{i\left(\xi_{j}+\xi_{k}\right)}\right)} \prod_{1 \leq j \leq n} \frac{\left(1-t_{1} e^{i \xi_{j}}\right)\left(1-t_{2} e^{i \xi_{j}}\right)}{1-e^{2 i \xi_{j}}}
$$


and

$$
\mathcal{N}_{\lambda}=\frac{(1-q)^{n}}{\left(t_{1} t_{2}\right)_{m_{0}(\lambda)} \prod_{l \geq 0}(q)_{m_{l}(\lambda)}} .
$$

Notice in this connection that one does not directly retrieve Macdonald's formula (17) by performing the limit $t_{3}, t_{4} \rightarrow 0$ in Venkateswaran's formula (5). Instead, the equivalence of the two formulas (for this specialization of the parameters) is not obvious and rather follows from the fact that both expressions represent the same polynomials of the form in equations (1) and $\Delta$ given by equation (3b) with $t_{3}=t_{4}=0[9,16]$. Since the expansion coefficients $C(w \xi)$ in equations (17) no longer depend on (the number of nonzero parts of) $\lambda$, in the present situation the coordinate Bethe ansatz form of the wave function $\phi_{\xi}$ (15) is seen to extend from the bulk sites $($ at $\ell>0)$ to the boundary site $($ at $\ell=0)$.

The actions of the annihilation and creation operators (9) on $f \in \ell^{2}\left(\Lambda_{n}, \mathcal{N}\right)$ now reduce to

$$
\left(\beta_{l} f\right)(\lambda)=f\left(\beta_{l}^{*} \lambda\right), \quad \lambda \in \Lambda_{n-1}
$$

with the convention that $\beta_{l} f=0$ if $n=0$, and

$$
\left(\beta_{l}^{*} f\right)(\lambda)=f\left(\beta_{l} \lambda\right)\left[m_{l}(\lambda)\right]\left(1-t_{1} t_{2} q^{m_{0}(\lambda)-1}\right)^{\delta_{l}}, \quad \lambda \in \Lambda_{n+1}
$$

with the convention that $\left(\beta_{l}^{*} f\right)(\lambda)=0$ if $m_{l}(\lambda)=0$. We thus arrive at an ultralocal twoparameter deformation of the $q$-boson field algebra at the boundary site $l=0$ represented by $\beta_{l}, \beta_{l}^{*}(18)$ and $N_{l}(10), l \in \mathbb{N}$ :

$$
\begin{aligned}
& \beta_{l} N_{k}=q^{\delta_{l-k}} N_{k} \beta_{l}, \quad \beta_{l}^{*} N_{k}=q^{-\delta_{l-k}} N_{k} \beta_{l}^{*}, \\
& \beta_{l}^{*} \beta_{l}=\frac{1-N_{l}}{1-q}\left(1-q^{-1} t_{1} t_{2} N_{0}\right)^{\delta_{l}}, \quad \beta_{l} \beta_{l}^{*}=\frac{1-q N_{l}}{1-q}\left(1-t_{1} t_{2} N_{0}\right)^{\delta_{l}},
\end{aligned}
$$

and

$$
\beta_{l} \beta_{k}=\beta_{k} \beta_{l}, \quad \beta_{l}^{*} \beta_{k}^{*}=\beta_{k}^{*} \beta_{l}^{*}, \quad \beta_{l} \beta_{k}^{*}=\beta_{k}^{*} \beta_{l}, \quad \beta_{l}^{*} \beta_{k}=\beta_{k} \beta_{l}^{*}
$$

if $l<k$. The corresponding $q$-boson Hamiltonian $H(12)$, with the reduction of the boundary potential $V\left(N_{0}, N_{1}\right)(14)$ given by

$$
V\left(N_{0}, N_{1}\right)=V\left(N_{0}\right):=\left(t_{1}+t_{2}\right)\left(\frac{1-N_{0}}{1-q}\right),
$$

acts on $f$ in the $n$-particle subspace $\ell^{2}\left(\Lambda_{n}, \mathcal{N}\right)$ via

$$
\begin{aligned}
\left(H_{n} f\right)(\lambda)= & \sum_{\substack{1 \leq j \leq n \\
\text { s.t. } \lambda+e_{j} \in \Lambda_{n}}} f\left(\lambda+e_{j}\right)\left[m_{\lambda_{j}}(\lambda)\right]\left(1-t_{1} t_{2} q^{m_{0}(\lambda)-1}\right)^{\delta_{\lambda_{j}}} \\
& +\sum_{\substack{1 \leq j \leq n \\
\text { s.t. } \lambda-e_{j} \in \Lambda_{n}}} f\left(\lambda-e_{j}\right)\left[m_{\lambda_{j}}(\lambda)\right]+f(\lambda)\left(t_{1}+t_{2}\right)\left[m_{0}(\lambda)\right] .
\end{aligned}
$$

The latter semi-infinite $q$-boson model with two-parameter boundary interactions was introduced and studied in more detail in [5].

Remark 3. When $q \rightarrow 0$ and $t_{r} \rightarrow 0(r=1, \ldots, 4)$, the action of our $n$-particle Hamiltonian $H_{n}$ on $f: \Lambda_{n} \rightarrow \mathbb{C}$ reduces to that of a discrete Laplacian

$$
\left(H_{n, 0} f\right)(\lambda)=\sum_{\substack{1 \leq j \leq n \\ \text { s.t. } \lambda+e_{j} \in \Lambda_{n}}} f\left(\lambda+e_{j}\right)+\sum_{\substack{1 \leq j \leq n \\ \text { s.t. } \lambda-e_{j} \in \Lambda_{n}}} f\left(\lambda-e_{j}\right)
$$


modeling a system of $n$ impenetrable bosons on $\mathbb{N}$. In [5, Section 5$]$ it was shown that the largetimes asymptotics of the $q$-boson dynamics generated by $H_{n}(19)$ is related to the impenetrable boson dynamics of $H_{n, 0}(3)$ via an $n$-particle scattering matrix of the form

$$
\mathcal{S}(\xi)=\prod_{1 \leq j<k \leq n} s\left(\xi_{j}-\xi_{k}\right) s\left(\xi_{j}+\xi_{k}\right) \prod_{1 \leq j \leq n} s_{0}\left(\xi_{j}\right)
$$

with

$$
s(x)=\frac{1-q e^{-i x}}{1-q e^{i x}} \quad \text { and } \quad s_{0}(x)=\frac{\left(1-t_{1} e^{-i x}\right)\left(1-t_{2} e^{-i x}\right)}{\left(1-t_{1} e^{i x}\right)\left(1-t_{2} e^{i x}\right)} .
$$

The discussion in [5, Section 5] applies verbatim to our more general Hamiltonian $H_{n}$ from Proposition 2 with $V\left(N_{0}, N_{1}\right)$ given by equation (14), upon replacing $s_{0}(x)$ (20b) by

$$
s_{0}(x)=\prod_{r=1}^{4} \frac{1-t_{r} e^{-i x}}{1-t_{r} e^{i x}} .
$$

This reveals that the $n$-particle scattering matrix of the model factorizes in two-particle bulk scattering matrices $s(\cdot)$ governed by a coupling parameter $q$ and one-particle boundary scattering matrices $s_{0}(\cdot)$ governed by coupling parameters $t_{1}, \ldots, t_{4}$.

\section{Acknowledgments}

We are grateful to Alexei Borodin and Ivan Corwin for helpful email exchanges and thank the referees for their constructive comments. This work was supported in part by the Fondo Nacional de Desarrollo Científico y Tecnológico (FONDECYT) Grants \# 1130226 and \# 11100315, and by

the Anillo ACT56 'Reticulados y Simetrías' financed by the Comisión Nacional de Investigación Científica y Tecnológica (CONICYT).

\section{References}

[1] Bogoliubov N.M., Izergin A.G., Kitanine N.A., Correlation functions for a strongly correlated boson system, Nuclear Phys. B 516 (1998), 501-528, solv-int/9710002.

[2] Borodin A., Corwin I., Petrov L., Sasamoto T., Spectral theory for the $q$-boson particle system, arXiv:1308.3475.

[3] van Diejen J.F., Properties of some families of hypergeometric orthogonal polynomials in several variables, Trans. Amer. Math. Soc. 351 (1999), 233-270, q-alg/9604004.

[4] van Diejen J.F., Emsiz E., Diagonalization of the infinite $q$-boson system, arXiv:1308.2237.

[5] van Diejen J.F., Emsiz E., The semi-infinite $q$-boson system with boundary interaction, Lett. Math. Phys., to appear, arXiv:1308.2242.

[6] Klimyk A., Schmüdgen K., Quantum groups and their representations, Texts and Monographs in Physics, Springer-Verlag, Berlin, 1997.

[7] Koornwinder T.H., Askey-Wilson polynomials for root systems of type $B C$, in Hypergeometric Functions on Domains of Positivity, Jack Polynomials, and Applications (Tampa, FL, 1991), Contemp. Math., Vol. 138, Amer. Math. Soc., Providence, RI, 1992, 189-204.

[8] Korff C., Cylindric versions of specialised Macdonald functions and a deformed Verlinde algebra, Comm. Math. Phys. 318 (2013), 173-246, arXiv:1110.6356.

[9] Macdonald I.G., Orthogonal polynomials associated with root systems, Sém. Lothar. Combin. 45 (2000), Art. B45a, 40 pages, math.QA/0011046.

[10] Macdonald I.G., Affine Hecke algebras and orthogonal polynomials, Cambridge Tracts in Mathematics, Vol. 157, Cambridge University Press, Cambridge, 2003. 
[11] Majid S., Foundations of quantum group theory, Cambridge University Press, Cambridge, 1995.

[12] Povolotsky A.M., On integrability of zero-range chipping models with factorized steady state, J. Phys. A: Math. Theor. 46 (2013), 465205, 25 pages, arXiv:1308.3250.

[13] Sasamoto T., Wadati M., Exact results for one-dimensional totally asymmetric diffusion models, J. Phys. A: Math. Gen. 31 (1998), 6057-6071.

[14] Takeyama Y., A discrete analogue of periodic delta Bose gas and affine Hecke algebra, arXiv:1209.2758.

[15] Tsilevich N.V., The quantum inverse scattering problem method for the $q$-boson model and symmetric functions, Funct. Anal. Appl. 40 (2006), 207-217, math-ph/0510073.

[16] Venkateswaran V., Symmetric and nonsymmetric Hall-Littlewood polynomials of type BC, arXiv:1209.2933. 